\title{
Deposition of Proton Conductive Oxide Thin films on a Porous Substrate for Fuel Cell Applications
}

\author{
Kiyoshi Uchiyama, Yuri Isse*, Yuichi Hori*, Takashi Nishida*, and Yukiharu Uraoka**** \\ Tsuruoka Nationatl College of Technology, Inooka Sawada 104, Tsuruoka, Yamagata 997-8511, Japan \\ Fax: 81-235-24-1840, e-mail: uchiyama@tsuruoka-nct.ac.jp \\ * Graduate School of Materials Science, Nara Institute of Science and Technology, \\ Takayama-cho 8916-5, Ikoma, Nara, 630-0192, Japan \\ ** CREST, Japan Science and Technology Agency, 4-1-8 Honcho, Kawaguchi,Saitama 332-0012, Japan
}

\begin{abstract}
An intermediate-temperature $\left(400-600{ }^{\circ} \mathrm{C}\right)$ operation of solid oxide fuel cells (SOFCs) is considered to be one of the solutions for reducing a SOFC apparatus cost. In order to achieve this, we proposed to use proton conductive thin films as an electrolyte. As a substrate is required a fuel gas permeability, porous substances are used as substrate, in general. However, this makes depositing solid electrolyte quite difficult. In this paper, we will mention our proposals for avoiding this difficulty. One of our proposals is a direct deposition on a polished porous substrate by liquid-delivery metal-organic chemical vapor deposition (LD-MOCVD). The other is a sol-gel deposition of SZYO thin films on Pd plated porous substrate. Based on these new SOFC structures, we believe we can obtain good SOFC operations in a near future.
\end{abstract}

Key words: Oxide, Fuel cell, Proton conductive, Thin films, Intermediate-temperature

\section{INTRODUCTION}

Solid oxide fuel cells (SOFCs) are considered to be one of the candidates for future energy sources. However, one of the issues to extend their range of applications is a reduction in operating temperature. Generally, SOFCs need a temperature above $800{ }^{\circ} \mathrm{C}$ to operate, which is too high to use typical metallic materials as SOFC components. As a consequence, ceramic materials and/or temperature resistant-metallic materials must be used, which makes the devices more expensive. Thus, lowering the operating temperature is the key for the SOFCs to extend their applications. For this purpose, intermediate-temperature (IT) $\left(400-600{ }^{\circ} \mathrm{C}\right)$ operations are required for SOFCs.

The reason why the conventional SOFCs need high operating temperature above $800{ }^{\circ} \mathrm{C}$ was to achieve enough reduction of their electrolyte resistance. Thus, many researches on lowering temperature are devoted to find new electrolyte materials with high conductivity at low temperature. However, it is still under investigation to obtain suitable new materials for IT operations $[1,2]$.

On the contrary, fabricating thinner electrolyte is quite effective to reduce the resistance of the electrolyte because the resistance of the electrolyte is proportional to its thickness. However, thinning the electrolyte requires a substrate to support it mechanically and the substrate must possess fuel gas permeability for SOFC operations. Thus, porous substances like porous ceramics are usually used as a substrate. However, it makes quite difficult depositing solid thin films on the substrate, especially depositing less than $1 \mu \mathrm{m}$-thick electrolytes.

In 2005, Ito et al. proposed to use a combination of the proton conductive oxide and a solid palladium (Pd) foil as a substrate for fabricating SOFCs. As a Pd possesses hydrogen permeability, they could avoid the difficulty in depositing electrolyte. They successfully deposited 0.7 $\mu \mathrm{m}$-thick proton conductive oxide, i.e. doped- $\mathrm{BaCeO}_{3}$, as the electrolyte on the Pd foil substrate by the pulsed laser deposition (PLD) method and achieved good SOFC operations as low as $400^{\circ} \mathrm{C}$ [3]. However, the cost of the $\mathrm{Pd}$ foil substrate makes their SOFC too expensive and not realistic.

In these years, we have been working to resolve this issue with using inexpensive porous stainless-steel (PSS) substrates. We have been proposing two kinds of SOFC fabrications. One is a direct deposition of proton conductive oxides on the porous substrate by metal-organic chemical vapor deposition (MOCVD). The other is to use Pd plated PSS as a substrate. We have successfully achieved solid Pd layer deposition with plating Pd on the PSS. This substrate still uses Pd, however, our proposal needs only several- $\mu \mathrm{m}$ (or less) thick Pd, which is quite small amount of Pd compared to using Pd foils as the substrate [3]. In both cases, we adopted Y-doped $\mathrm{SrZrO}_{3}(\mathrm{SZYO})$ as a proton conductive electrolyte because it possesses chemical stability with relatively high conductivities [4]. In this paper, we will review our recent results by way of two kinds of depositions for SOFCs.

\section{EXPERIMENTAL}

For an MOCVD, we adopted liquid delivery (LD)-MOCVD technique which was used for $(\mathrm{Pb}, \mathrm{La})(\mathrm{Ti}, \mathrm{Zr}) \mathrm{O}_{3} \quad$ (PLZT) thin film depositions[4]. MOCVD precursors used in this deposition were $\mathrm{Sr}(\mathrm{METHD})_{2}, \quad \mathrm{Zr}(\mathrm{METHD})_{4}, \quad$ and $\mathrm{Y}(\mathrm{EDMMD})_{3}$ (ADEKA Corp.), which were dissolved in ethylcyclohexane $(\mathrm{ECH})$ at concentrations of $0.1,0.1$, and $0.01 \mathrm{M}$, respectively. (METHD and EDMMD denote the abbreviated notations of ligands of 
1-(2-methoxyethoxy)-2,2,6,6-tetramethyl-3,5-heptanedio nate and 6-ethyl-2,2-dimethyl-3,5-decanedionate; respectively.) These dissolved precursors were delivered individually at controlled flow rates by liquid mass flow controllers (L-MFCs) and were misted and vaporized in a vaporization chamber. The vaporized precursors and $\mathrm{ECH}$ were then introduced into the deposition chamber with an $\mathrm{O}_{2}$ gas to deposit the oxide thin films on the substrate. The atomic ratio of $\mathrm{Y} / \mathrm{Sr}$ in the SZYO films was chosen as $0.05 / 0.95$. The detailed LD-MOCVD technique is described elsewhere $[5,6]$. Typical deposition conditions are summarized in Table I.

Table I Typical deposition conditions of LD-MOCVD \begin{tabular}{l|l}
\hline Carrier gas $\left(\mathrm{N}_{2}\right)$ flow $(\mathrm{sccm})$ & 300 \\
\hline
\end{tabular}

\begin{tabular}{l|l}
\hline Oxidation gas $\left(\mathrm{O}_{2}\right)$ flow $(\mathrm{sccm})$ & $1000-3000$ \\
\hline Deposition temperature $\left({ }^{\circ} \mathrm{C}\right)$ & 550,600 \\
\hline Substrates & $\begin{array}{c}\mathrm{Pt} / \mathrm{SiO}_{2} / \mathrm{Si} \text {, porous } \\
\text { stainless-steel }\end{array}$ \\
\hline Deposition time $(\mathrm{min})$ & 30,45 \\
\hline Typical film thickness $(\mathrm{nm})$ & 200 \\
\hline
\end{tabular}

On the other hand, the deposition of the SZYO thin film on Pd plated PSS substrates was performed by a sol-gel method. The reason why the sol-gel method was chosen is to reduce an oxidization of Pd during deposition. When the MOCVD was performed, Pd was easy to be oxidized during depositions. In addition, we found that the sol-gel process can deposit high quality PLZT thin films when its conditions were optimized $[7,8]$ and considered it might also be applicable for high quality SZYO depositions.

The typical deposition procedure of the sol-gel method was shown in Fig. 1, which is almost the same as typical deposition process of PLZT thin films. The spin-coating of sol-gel precursor to the pyrolysis of precursor process were repeated several times to have desired thickness of the films. Detailed information of the deposition conditions are described in ref [9].

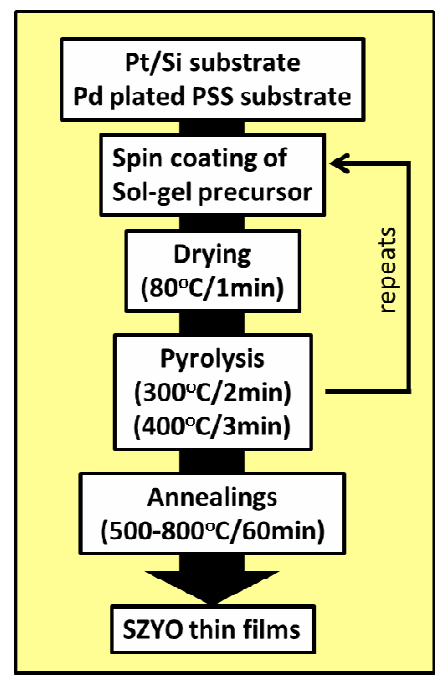

Fig. 1 Typical sol-gel process of SZYO depositions [9]

The crystallinity of the deposited films was evaluated by the $\theta-2 \theta$ method using X-ray diffraction (XRD). In general, the intensity of the diffraction of films with the same crystal structure and thickness reflects their crystallinity. In our experiment, we chose the (121) and (200) peaks of SZYO, which are very close to each other and cannot be distinguished, for evaluating the film crystallinity because they showed the highest intensity among the diffraction peaks. Cross-sectional and surface images of the films were obtained by scanning electron microscopy (SEM). The gas leakage through the electrolyte was measured using a pressurized He gas. As the deposition temperature of $600{ }^{\circ} \mathrm{C}$ was the maximum temperature obtainable using our apparatus, we examined deposition temperatures of 550 and $600{ }^{\circ} \mathrm{C}$ to examine the effect of deposition temperature on the crystallinity of the films.

We also examined the gas barrier property of the deposited films using a pressurized He gas. Fig. 2 is the schematic of the measurement apparatus. A pressurized He at 2 atms was applied to one side of the film and the other side was left opened to the atmosphere. Then, we measured the period required for its pressure drop from 2 to $1 \mathrm{~atm}$, which represents the degree of leakage. In addition, we also measured the He concentration of the opened side of the film to confirm the existence of $\mathrm{He}$ leakage through the films by using a He detector.

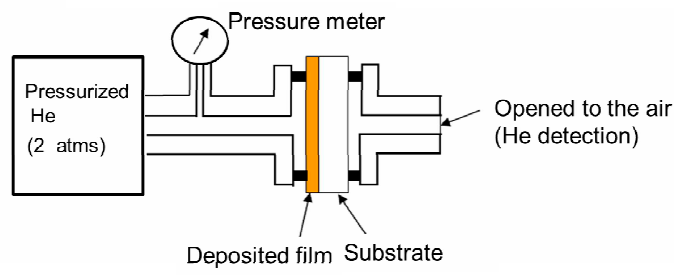

Fig.2 Schematic of gas leak measurement apparatus

\section{RESULTS AND DISCUSSION}

3.1 Direct deposition on porous substrate by LD-MOCVD

Fig. 3 shows the SEM images of SZYO thin films directly deposited on the porous stainless-steel (PSS) substrates by LD-MOCVD [9]. The substrates that used in the depositions were as-prepared and polished ones. As can be seen in Fig. 2, as-prepared substrate has large roughness more than $10 \mu \mathrm{m}$. In turn, the polished substrate has quite smooth surface but has several $\mu \mathrm{m}$ size pores on it.

As can be seen in Fig. 3, no film formation was observed for the deposition on as-prepared substrate. On the contrary, sold film formation was observed for the deposition on the polished PSS substrate. The film was fully crystallized with as-deposited state and no post annealing was needed for crystallization. In addition, it was deposited even on the pores with bridging them. The film was solid and defect-less thin films with the thickness of $200 \mathrm{~nm}$. In general, it is quite difficult to deposit solid thin films on this kind of large pores, however, the combination of polished substrate and MOCVD has brought a solid film deposition. The reason why this type of solid film growth was possible on the porous substrate remains unclear, however, the in-plane growth at the edge of the pores might be dominant. 


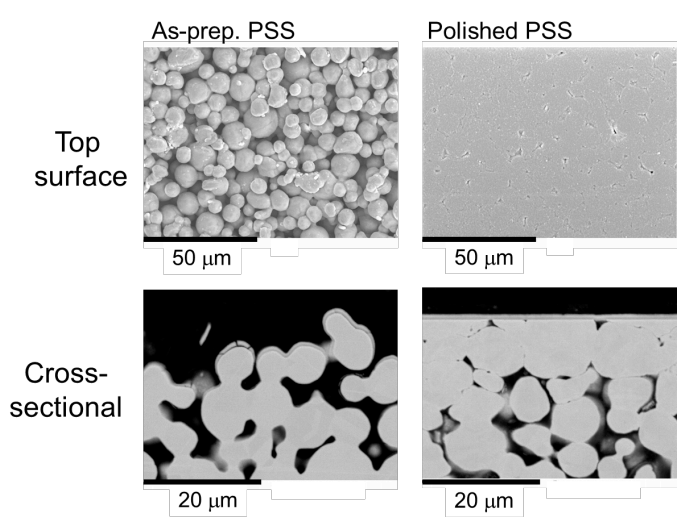

Fig. 3 Top \& Cross-sectional SEM images of MOCVD deposited SZYO thin films on as-prepared (as-prep.) and polished PSS substrates. [6]

This result suggests that an SOFC with a 200-nm-thick electrolyte can be fabricated on the porous substrate. To confirm this, we examined the gas barrier property of the film using pressurized He gas (Fig.2). Pressurized He at 2 atms was applied to one side of the film and the other side was left open to the atmosphere; we then measured the period required for its pressure to decrease from 2 to 1 atm. However, no pressure decrease was observed up to $1500 \mathrm{sec}$. for this sample. In addition, no He leakage was observed at the side opened to the atmosphere by a He detector. This means that the film has a good gas barrier property and lacks defects, which is suitable for use in SOFCs.

We examined to fabricate the SOFC single cell, however, we met a short-circuiting of the electrolyte during top electrode fabrication. To avoid this, we have been developing the low temperature electrode fabrication process using a sol-gel method.

3.2 Sol-gel deposited SZYO thin films on Pd plated PSS substrate

The other approach for the SOFC fabrication with thin film electrolyte is to use Pd plated PSS as a substrate. As shown in our former paper, we have successfully fabricated solid Pd thin film on PSS by plating with no gas leakage [9]. As Pd has hydrogen permeability, this substrate can supply hydrogen as a fuel gas, which means no pores are needed on the top of the substrate. Thus, we can use conventional deposition methods of oxide thin films, like sputtering, PLD, sol-gel, etc., for SOFC fabrication by using this substrate. We chose sol-gel method because it is cheaper thin film fabrication process than MOCVD, which will be realistic process for actual device fabrications. In addition, we considered that sol-gel process can reduce Pd oxidization during film depositions when its conditions are optimized. The atomic ratio of $\mathrm{Y} / \mathrm{Sr}$ in $\mathrm{SZYO}$ was chosen as $0.2 / 0.8$ to obtain higher proton conductivity.

Fig.4 shows the XRD patterns of sol-gel derived SZYO thin films annealed at various temperatures in air deposited on $\mathrm{Pt} / \mathrm{SiO}_{\mathrm{x}} / \mathrm{Si}$ substrates [9].

The as-prepared sample was non-crystallized and samples annealed below $550{ }^{\circ} \mathrm{C}$ were not crystalized neither. In turn, annealings above $600{ }^{\circ} \mathrm{C}$ gave the crystallization of the SZYO films and no significant change in XRD patterns was observed, which suggests annealing at $600{ }^{\circ} \mathrm{C}$ is enough to have the crystallization of the film. This is quite important for PSS not to be deformed and warped during the annealing of SZYO.

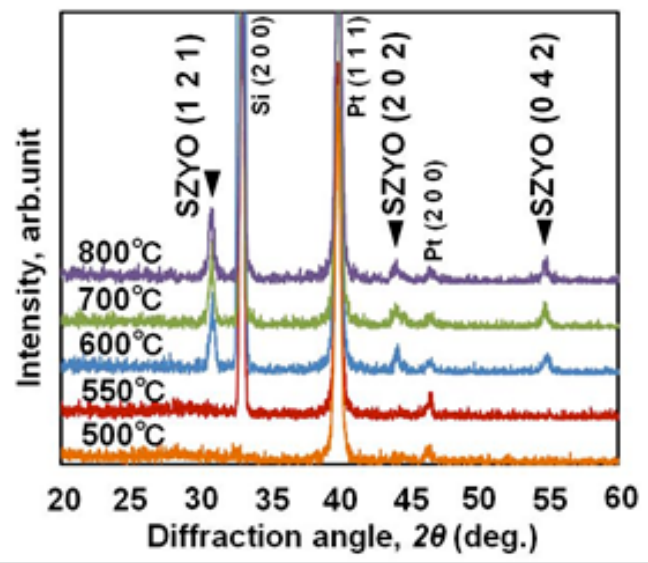

Fig.4 XRD patterns of the SZYO films annealed in air at various temperatures.[9]

Fig. 5 shows a cross-sectional SEM image of the SZYO thin film deposited on Pd plated PSS after the crystallization [9]. As can be seen in Fig. 5, smooth interface was observed at $\mathrm{SZYO} / \mathrm{Pd}$ interfaces, which is considered to be suitable for SOFC fabrications. However, XRD analysis showed the existence of PdO, which suggests some kind of oxidization is occurred in the Pd layer. Actually, we met the shorting of the electrolyte in this SOFC structure and could not measure SOFC performances. This shorting might come from the oxidization of the Pd as well as the higher temperature of electrode fabrication, as mentioned in 3.1. Thus, we are working to suppress a Pd oxidation by annealing in vacuum as well as lowering electrode fabrication temperature.

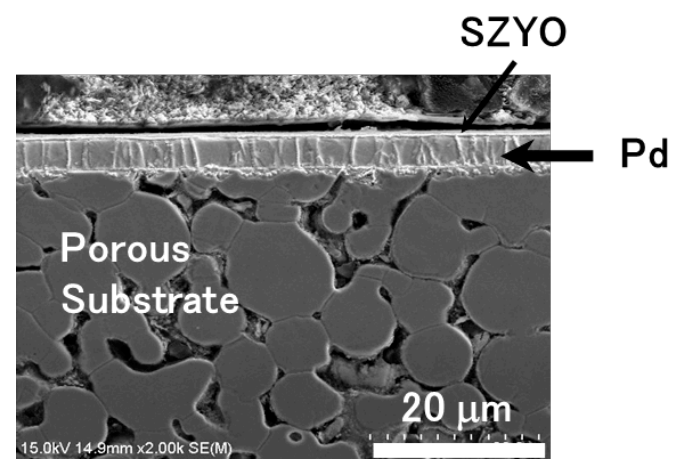

Fig.5 Cross-sectional SEM image of SZYO thin film deposited on Pd plated porous substrate (PSS) by sol-gel method.)[9]

\section{CONCLUSIONS}

To obtain lower operation temperature of the SOFCs, we examined two kinds of SOFC structures based on the proton conductive thin films.

One is a direct deposition of proton conductive 
(SZYO) thin films on the polished PSS substrates by LD-MOCVD. The other is a sol-gel deposition of SZYO on the Pd plated PSS substrate. Both approaches gave a good solid SZYO thin film depositions with no significant defects, which is applicable for SOFC fabrications. However, we still need to solve other issues for actual SOFC fabrications. One is a lowering process temperature of the top electrode. The other is a suppressing the oxidation of the $\mathrm{Pd}$ layer during processes. We are working these issues to have actual SOFC cell fabrications. We believe we would have good SOFC operations based on our proposed SOFC structures.

\section{ACCKNOLEDGEMENTS}

We would like than to Drs. K. Yanagimoto and T. Kariya of Sanyo Special Steel, Co. Ltd. for their kindly assistance. We also like thank to Prof. H. Suematsu of Nagaoka University of Technology and Prof H. Funakubo of Tokyo Institute of Technology for the measurements of the samples.

This work is partially supported by Nagaoka University of Technology and Tsuruoka National College of Technology.

\section{REFERENCES}

[1] K. Eguchi, T. Setoguchi, T. Inoue and H. Arai, Solid State Ionics, 52, 165 (1992)
[2] O. Yamamoto, Y. Arati, Y. Takeda, N. Imanishi, Y. Mizutani, M. Kawai and Y. Nakamura, Solid State Ionics, 79, 137 (1995).

[3] N. Ito, M. Iijima, K. Kimura, and S. Iguchi, J. Power Sources, 152, 200 (2005).

[4] T. Higuchi, T. Tsukamoto, S. Yamaguchi, N. Sata, K. Hiramoto, M. Ishigame, and S. Shin, Jpn. J. Appl. Phys., 41 (2002) 6440.

[5] Y. Otani, K. Uchiyama, S. Okamura and T. Shiosaki, Integr. Ferroelectr., 81, pp. 261-270 (2006).

[6] K. Uchiyama, H. Sakairi, T. Shiosaki, T. Kariya, H. Ikeda, K. Yanagimoto, Y.Yamada, A. Nakayama, Jpn. J. Appl. Phys., 48, 070205(2009). [7] K. Uchiyama, A. Kasamatsu, Y. Otani and T. Shiosaki, Jpn. J. Appl. Phys., 46, L244 (2007). [8]K. Uchiyama, T. Shiosaki, T. Kosaka, A. Kasamatsu and M. Echizen, Ceramics International, 34, 979(2008).

[9] K. Uchiyama, Y. Isse, Y. Hori, T. Nishida, Y. Uraoka, T. Kariya, and K. Yanagimoto, Proc. 6th Thin-Films Materials and Devices Meeting, 100228102-1-4 (Feb, 2010)

(Received Janurary 31, 2011; Accepted May 23, 2011) 